\title{
Determination of the Effective Refractive Index of Various Single Mode Fibres for Fibre Bragg Grating Sensor Applications
}

Jülich, Florian and Roths, Johannes*

Munich University of Applied Sciences

Lothstr. 34, D-80335 Munich, Germany

\begin{abstract}
A method for determining the effective refractive index $\left(n_{\text {eff }}\right)$ of optical fibres with high accuracy is presented. The measurement principle is based on the inscription process of fibre Bragg gratings (FBG) into the optical fibres. Results of refractive index measurements at $\sim 1535 \mathrm{~nm}$ wavelength on standard telecommunication and speciality, photosensitive optical fibres are presented. The measured values of $n_{\text {eff }}$ correlate with $\mathrm{GeO}_{2}$ dopant concentration inside the cores of the fibres. This measurement technique can have applications in process control for optical fibre manufacturing and in FBG production technology.
\end{abstract}

Keywords: optical fibre, fibre Bragg gratings, effective refractive index, photosensitivity

\section{INTRODUCTION}

Since the first investigation of photosensitivity by Hill and co workers ${ }^{[1]}$, the formation of fibre Bragg gratings (FBG) has been demonstrated in a large variety of different types of optical fibres with specific characteristics. FBGs have become an important filter device for optical telecommunication applications and a new type of optical fibre sensor ${ }^{[2-4]}$.

The types of optical fibres which are currently used are for example photosensitive fibres, hydrogenated standard telecom fibres, cladding mode suppressed or even polarisation maintaining fibres. In the socalled photosensitive fibres, the cores of the fibres are highly doped with $\mathrm{GeO}_{2}$ (or other materials), in order to make them more sensitive to the UV-light induced increase of the refractive index, which is commonly known as photosensitivity. The concentration of the dopant also strongly determines the core refractive index (and thus the effective refractive index $\left(n_{\text {eff }}\right)$ ) of the unexposed fibre. For a stable and controlled fabrication of FBGs it is necessary to know precisely the effective refractive index at the working wavelength. This parameter is responsible for the grating wavelength and mostly not provided with high accuracy by the manufacturers of the fibres.

\section{THEORY}

An optical fibre Bragg grating is a permanent, periodic variation of the refractive index within the core of an optical fibre ${ }^{[3]}$. Due to the photosensitivity of optical fibres, these structures are generated by exposing optical fibres to a UV interference pattern. When the phase mask technique ${ }^{[5]}$ is employed to generate the interference pattern, its period $\Lambda$ (and thus the period of the FBG) is directly related to the period of the phase mask $\Lambda_{P M}$, by

$$
\Lambda=\frac{\Lambda_{P M}}{2}
$$

It is worth to note, that $\Lambda$ does not depend on the wavelength of the UV light. The UV exposure creates a periodic index variation with amplitude $\Delta n$ and pitch $\Lambda$ that extends over a certain length $L$ of the fibre (see Figure 1). The change of the refractive index along the region of a fibre Bragg grating can be divided into a DC offset (mean amplitude) and an AC part (sinusoidal variation). The refractive index of a fibre that was exposed to a certain amount of UV light dose $d$ can be approximately described as a function of position $(z)$ and UV dose $(d)$,

$$
n_{\text {eff }}(z, d)=n_{\text {eff }}+\Delta n_{U V}(z, d)=n_{\text {eff }}+\left(\Delta n_{D C}(d)+\Delta n_{A C}(d) \cdot \sin \left(\frac{2 \pi}{\Lambda} \cdot z\right)\right) .
$$

For most wavelengths, optical waves propagating along the fibre are nearly unaffected by the grating, but for a certain wavelength such a periodic structure acts as a Bragg reflector with a pronounced reflectivity. This wavelength, the so-called Bragg wavelength $\lambda_{B}$, satisfies the condition ${ }^{[2,3]}$.

$$
\lambda_{B}=2 \cdot \overline{n_{e f f}} \cdot \Lambda
$$


where $\overline{n_{\text {eff }}}$ is the mean effective refractive index at the location of the grating. The basic idea of this method is, that when the pitch of the phase mask, $\Lambda_{P M}$, is known (by microscopic observation for example), and the resulting Bragg wavelength $\lambda_{B}$ is measured, the mean refractive index of the optical fibre $\overline{n_{\text {eff }}}$, can be determined.

A change in the grating period $\Lambda$ and/or in the effective refractive index $n_{\text {eff }}$, due to external influences, will result in a shift of the reflected Bragg wavelength. The measurement of this wavelength shift, as a consequence of external influences, is the basis for optical FBG sensors. The grating period and the

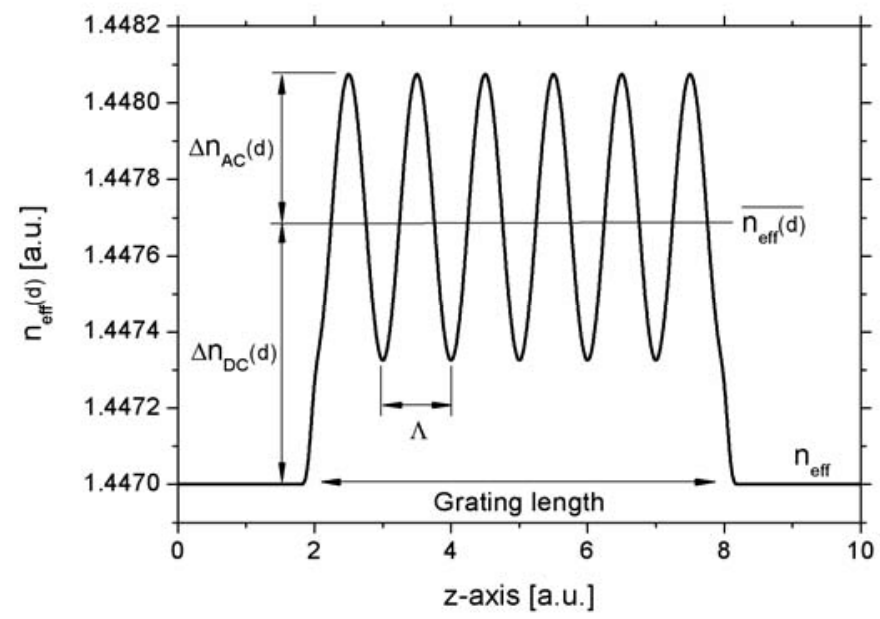

Fig. 1: Refractive index profile of a fibre Bragg grating. refractive index depend directly on strain $(\varepsilon)$ and temperature $(T)$, which makes them the primary measurands for FBG sensors. The present study was performed at nearly constant (room) temperature $T=\left(20^{\circ} \mathrm{C} \pm 2 \mathrm{~K}\right)$. Both, the grating pitch $\Lambda$ and the mean refractive index $\overline{n_{\text {eff }}}$ depend on strain, and the shift of the Bragg wavelength at constant temperature can be approximated by ${ }^{[4]}$

$$
\Delta \lambda(\varepsilon)=2 \cdot\left[\frac{\partial \Lambda}{\partial \varepsilon} \cdot n_{e f f}+\frac{\partial n_{e f f}}{\partial \varepsilon} \cdot \Lambda\right] \cdot \varepsilon=\lambda_{B}(0) \cdot\left(1-p_{e}\right) \cdot \varepsilon,
$$

where $p_{e}$ is the effective photo-elastic coefficient of an optical fibre, which was determined earlier for some of the fibres employed here ${ }^{[6]}$.

Here, we focus on the effective refractive index of the pristine fibre; therefore two further aspects have to be considered. First, the inscription process leads to an increase in the mean effective refractive index, due to the dc-component, $\Delta n_{\mathrm{DC}}$, of the UV-induced index change. Therefore, the measured Bragg wavelengths have to be extrapolated to the condition of zero UV dose $(d=0)$, leading to the so-called design wavelength

$$
\lambda_{D}=\lambda_{B}(d=0)=\overline{n_{e f f}}(d=0) \cdot 2 \cdot \Lambda=n_{e f f} \cdot \Lambda_{P M}
$$

which is directly related to $n_{\text {eff }}$ because of $\lim _{d \rightarrow 0}\left(\overline{n_{\text {eff }}}(d)\right)=n_{\text {eff }}$.

Second, when the optical fibre is installed in the FBG inscription device, it is fixed with a certain stress between two fibre clamps. This mechanical stress induces a slight decrease of the refractive index due to the photo-elastic effect. This refractive index change is described by the effective photo-elastic coefficient $p_{e}$, as was still mentioned above,

$$
\Delta n(\varepsilon)=n(\varepsilon=0)-n(\varepsilon)=n(\varepsilon=0) \cdot p_{e} \cdot \varepsilon
$$

For the sake of simplicity, in Eq. (6) $n$ is directly replaced by the mean effective refractive index $\overline{n_{\text {eff }}}$ and by combination of Eq. (6) and Eq. (5), the mean refractive index at zero strain $(\varepsilon=0)$ and at zero UV dose $(d=0)$ is obtained as

$$
n_{e f f}(\varepsilon=0, d=0)=\frac{n_{e f f}(d=0)}{1-p_{e} \cdot \varepsilon}=\frac{\lambda_{D} / \Lambda_{P M}}{1-p_{e} \cdot\left(\frac{\lambda_{B, s}-\lambda_{B, u}}{\lambda_{B, u}\left(1-p_{e}\right)}\right)} \cong \frac{\lambda_{D} / \Lambda_{P M}}{1-p_{e} \cdot\left(\frac{\Delta \lambda}{\lambda_{D}\left(1-p_{e}\right)}\right)} .
$$

\section{EXPERIMENTAL}

Our intention in this study was to compare the effective refractive indices of optical fibres which differ in material composition and core diameter. In this investigation, we choose standard optical fibres (SMF-28, Siecor, Corning), a photosensitive fibre with low NA (GF1B; Nufern) and highly Ge-doped photosensitive 
fibres (F86, F89-18, PR2008, SF 10-2007 (IPHT; Jena)). The main parameters of the fibres used in this investigation are compiled in Table 1. The values of the diameters of the core and cladding were measured with a microscope and the coating diameters were obtained with a micrometer. For FBG generation, we used our in-house FBG inscription facility ${ }^{[7]}$, which is based on an Excimer laser (ExciStar S200, TUI/Coherent) and a phase mask (O/E Land) (see Figure 2). Before exposure, the fibre coatings were removed with a standard stripping tool.

Tab. 1: Optical fibres investigated in the present study

\begin{tabular}{|c|c|c|c|c|}
\hline $\begin{array}{l}\text { Fibre type I } \\
\text { Manufacturer }\end{array}$ & $\begin{array}{l}\text { coating } \\
\text { material }\end{array}$ & $\begin{array}{c}\text { Diameters: core / } \\
\text { cladding / coating } \\
{[\mu \mathrm{m}]}\end{array}$ & $\begin{array}{l}\text { Core dopant I } \\
\text { concentration }\end{array}$ & Speciality \\
\hline $\begin{array}{l}\text { SMF } 28(1) / \\
\text { Siecor }\end{array}$ & Acrylate & 9.2 / 125 / 232 & $\mathrm{GeO}_{2} / \sim 3.5 \%$ & Standard telecom \\
\hline $\begin{array}{l}\text { SMF } 28(2) / \\
\text { Corning }\end{array}$ & Acrylate & 8.2 / 124 / 227 & $\mathrm{GeO}_{2} / \sim 3.5 \%$ & Standard telecom \\
\hline GF1B / Nufern & Acrylate & 9.9 / 125 / 232 & $\mathrm{GeO}_{2} / \sim 5 \%$ & $\begin{array}{c}\text { Photosensitive, low } \\
\text { NA }\end{array}$ \\
\hline F86 / IPHT & Acrylate & $4.9 / 125 / 220$ & $\mathrm{GeO}_{2} / \sim 16 \%$ & Photosensitive \\
\hline F89-18 / IPHT & ORMOCERE $^{[8]}$ & $5.0 / 126 / 182$ & $\mathrm{GeO}_{2} / \sim 16 \%$ & Photosensitive \\
\hline PR2008 / IPHT & Acrylate & $4.4 / 125 / 219$ & $\mathrm{GeO}_{2} / \sim 16 \%$ & Photosensitive \\
\hline $\begin{array}{l}\text { SF } 10-2007 / \\
\text { IPHT }\end{array}$ & ORMOCERE $^{[8]}$ & $4.0 / 124 / 182$ & $\mathrm{GeO}_{2} / \sim 16 \%$ & $\begin{array}{c}\text { Photosensitive, low } \\
\text { loss }\end{array}$ \\
\hline
\end{tabular}

The output power of the Excimer laser was focused by a cylindrical lens and the optical fibre was placed close to the focus line of the laser beam within the interference pattern that was created by the phase mask (s. Figure 3). The average laser power was measured before and after each inscription process, using a power meter (FieldMaster, Coherent). Dividing the average power by the pulse repetition frequency $(P R F=1001 / \mathrm{s})$, gives an estimate of the mean UV pulse energy. The reflection spectrum of the forming FBG was observed during the inscription process with an optical spectrum analyser (OSA) (Q8384, Advantest) in combination with an ASE-light source (NetTest) and a 2x2 coupler. The OSA had a specified offset and linearity error of $10 \mathrm{pm}$ in the spectral range between $1530 \mathrm{~nm}$ to $1570 \mathrm{~nm}$. But during short time periods (typ. $30 \mathrm{~min}$ ) after the built-in calibration procedure had been performed, the OSA showed significantly better accuracy values. The accuracy in the spectral range of the measurement $(1533-1543 \mathrm{~nm})$ was experimentally verified by measuring the absorption lines of Acetylene $\left({ }^{12} \mathrm{C}_{2} \mathrm{H}_{2}\right)$, as shown in Fig. 4, and by comparing the fitted centre wavelengths with the NIST Certificate SRM $2517 \mathrm{a}^{[9]}$. The result is presented in Fig. 5 as a function of the measurement difference $\left(\lambda_{\text {measured }}-\lambda_{\text {NIST }}\right)$ versus wavelength, showing an accuracy of $\pm 1.5 \mathrm{pm}$.

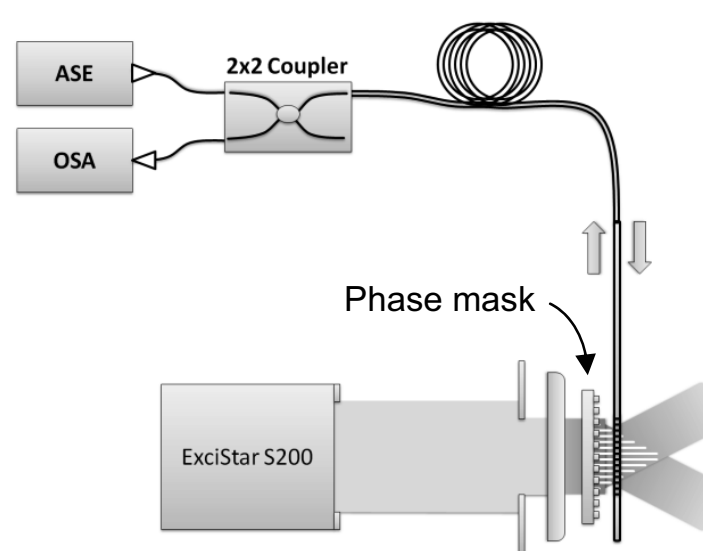

Fig. 2: Schematic of the experimental set-up.

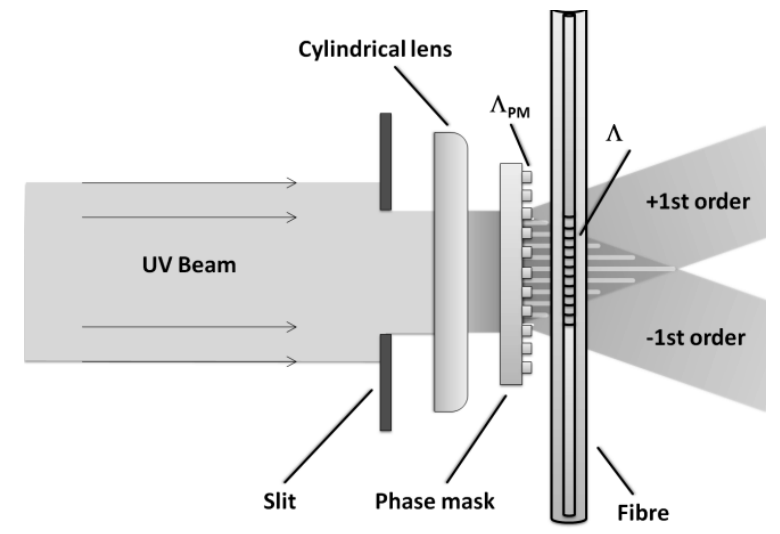

Fig. 3: Phase mask inscription schematic 


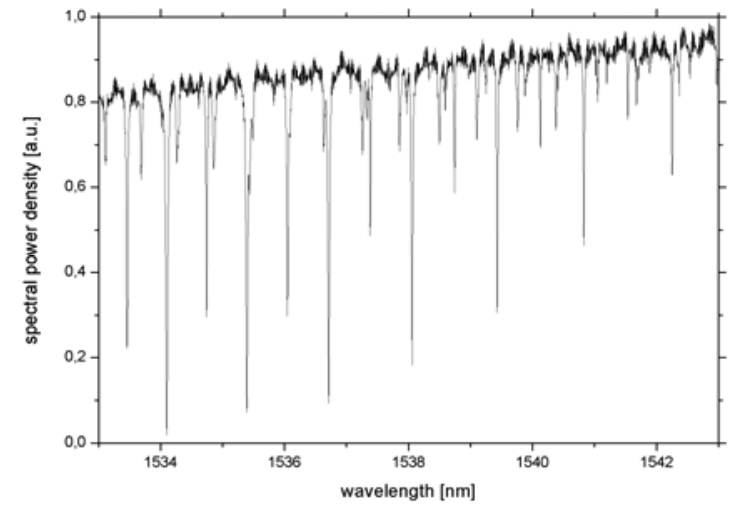

Fig. 4: Absorption spectra of Acetylene $\left({ }^{12} \mathrm{C}_{2} \mathrm{H}_{2}\right)$.

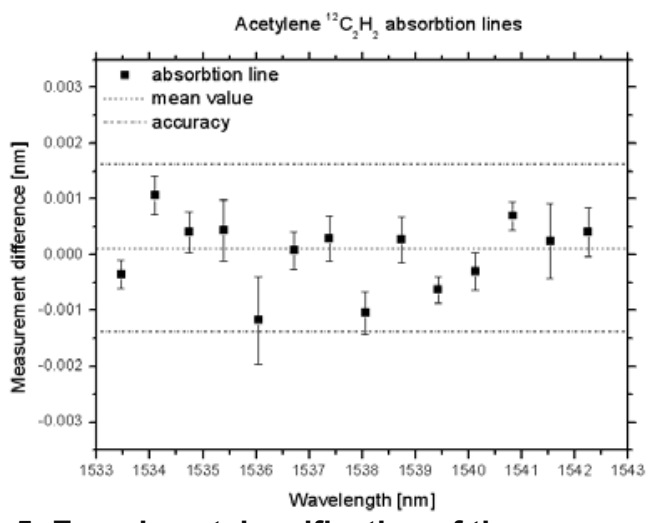

Fig. 5: Experimental verification of the accuracy of the optical spectrum analyser.

As an example for the inscription of a fibre Bragg grating. Fig. 6 shows the formation of the Bragg reflection peak with increasing quantity of laser shots. For the obtained data, the centre wavelength of the generated FBG ( $\lambda_{B}[\mathrm{~nm}]$ ) was determined with high precision by fitting a second order polynomial function to the peak of the FBG reflection spectrum. The peaks of the spectral shape functions were well approximated by quadratic functions and the centre wavelengths were determined with high precision (s. Fig. 6). The UV-light dose $d$ [J], to which the fibre was exposed to, can be assumed to be proportional to the mean UV pulse energy, multiplied by the number of pulses. In Figure 7 , the Bragg wavelength $\left(\lambda_{B}\right)$ is plotted as a function of the exposure dose $(d)$. This graph, the so called FBG inscription curve, shows the typical behaviour of a non-linear increase of $\lambda_{\mathrm{B}}$ as a function of UV dose $d$. In order to determine the design wavelength, i. e. to extrapolate the Bragg wavelength $\lambda_{\mathrm{B}}(d)$ to its zero UV dose $(d=0)$, a linear regression calculation was applied to the first 10 data points and the ordinate intersection of the fitted linear function is taken as $\lambda_{d}$, as is shown in the inset of Figure 7. At the end of each inscription procedure, the fibre was released from its fixations and the Bragg wavelength of the unstressed fibre was measured again. As can be seen in Figure 7, the unstressed fibre showed a significantly lower value and the corresponding wavelength change $\Delta \lambda(\varepsilon)=\lambda_{B, s}-\lambda_{B, u}$ between the final value in strained $\left(\lambda_{B, s}\right)$ and unstrained $\left(\lambda_{B, u}\right)$ condition was used to determine the strain $\varepsilon$ of the fibre when it had been installed in the FBG inscription facility.

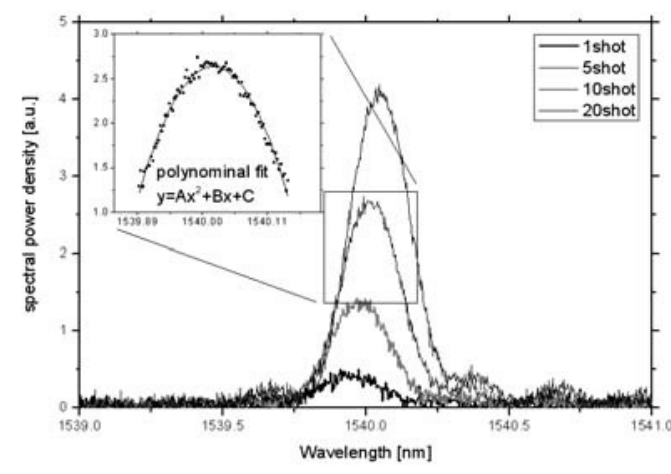

Fig. 6: Formation of a FBG during inscription. Inset: Example of a polynomial fit on the spectral shape function of a fibre Bragg grating

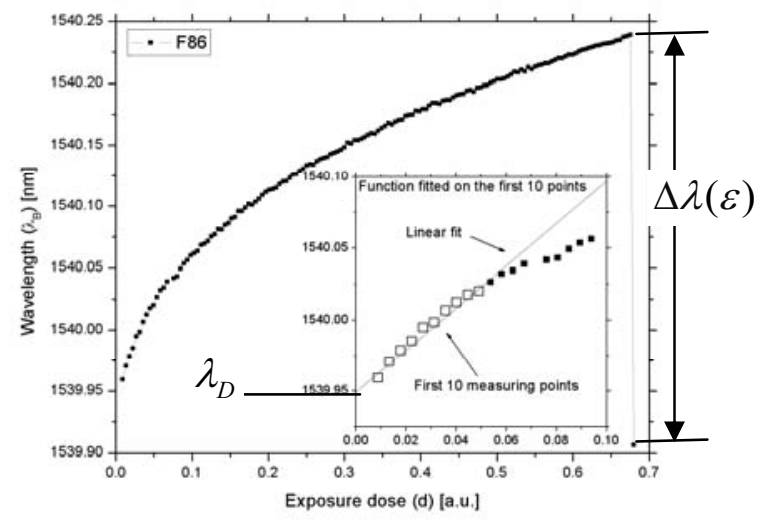

Fig. 7: Inscription of a highly photosensitive fibre (F86). Inset: Example of a linear fit along the first 10 measurement points of the inscription curve

\section{RESULT AND DISCUSSION}

For all optical fibres investigated in this study, the design wavelengths $\lambda_{D}$ and strain induced wavelength changes $\Delta \lambda$ were evaluated from the FBG inscription curves as described above. The obtained values are compiled in Table 2. The uncertainty in $\lambda_{D}$ was estimated to be $\sigma_{\lambda_{D}}=20 \mathrm{pm}$, which is mainly attributed to the fact, that here a non-linear relationship was approximated by a linear function. Care was taken that the first datapoint of the FBG inscription curve was taken at the smallest possible UV dose, which was given by the condition, that the FBG reflection line sphape was still visible $(S N R>1)$ in the 
spectrum. The wavelength accuracy and the linearity error of the OSA can be neglected relative to the uncertainty in the linear extrapolation of the inscription curve. The mean effective refractive indices were calculated according to Eq. (7), using a value for the effective photo-elastic coefficient $p_{\mathrm{e}}$ of $(0.205 \pm 0.005)$ for all fibres ${ }^{[6]}$. The phase mask grating had a pitchvalue of $\Lambda_{\mathrm{PM}}=(1060.0 \mu \mathrm{m} \pm 0.1 \mu \mathrm{m})$. This manufacturer specified accuracy is the dominant contribution to the overall uncertainty in the determination of $n_{\text {eff }}$. Additional uncertainties were the tilt of the optical fibre and laser beam relative to the phase mask, $\left(\sigma\left(n_{\text {eff }}\right) /\left.n_{\text {eff }}\right|_{\text {alignment }} \cong 3 \cdot 10^{-6}\right.$, and

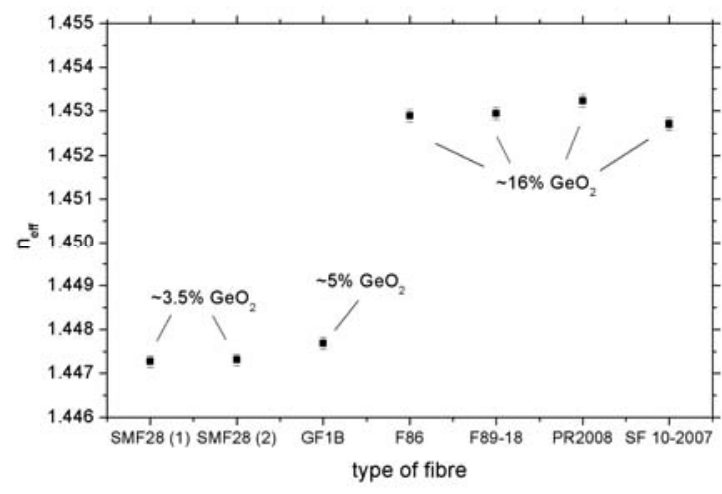

Fig. 8: effective refractive indices with their total uncertainties temperature drifts during the measurements, $\sigma\left(n_{\text {eff }}\right) /\left.n_{\text {eff }}\right|_{\text {Temperature }} \cong 1.3 \cdot 10^{-5}$. The total uncertainty in determining $n_{\text {eff }}$ at the corresponding wavelengths can be estimated to be $\sigma\left(n_{\text {eff }}\right) /\left.n_{\text {eff }}\right|_{\text {total }} \cong 1 \cdot 10^{-4}$. The measured effective refractive indices with their total uncertainties of all fibres used in this study are depicted in Figure 8 and shown in Table 2. The values for the concentration of $\mathrm{GeO}_{2}$ in the fibres cores, as provided by the manufacturers were given in Figure 8, too.

Tab. 2: Design wavelengths and mean effective refractive indices with their total uncertainties

\begin{tabular}{|c|c|c|c|}
\hline Fibre Type & $\begin{array}{c}\text { Design } \\
\text { wavelength } \lambda_{\mathrm{D}} \\
{[\mathrm{nm}]}\end{array}$ & $\begin{array}{c}\text { Strain induced } \\
\text { wavelength change } \\
\Delta \lambda,[\mathrm{nm}]\end{array}$ & $\begin{array}{c}\text { effective refractive } \\
\text { index } n_{\text {eff }}\end{array}$ \\
\hline SMF 28 (1) & $1534.014 \pm 0.020$ & $0.385 \pm 0.002$ & $1.44728 \pm 0.00014$ \\
\hline SMF 28 (2) & $1534.064 \pm 0.020$ & $0.367 \pm 0.002$ & $1.44732 \pm 0.00014$ \\
\hline GF1B & $1534.835 \pm 0.020$ & $0.173 \pm 0.002$ & $1.44800 \pm 0.00014$ \\
\hline F86 & $1539.954 \pm 0.020$ & $0.332 \pm 0.002$ & $1.45287 \pm 0.00014$ \\
\hline F89-18 & $1540.055 \pm 0.020$ & $0.240 \pm 0.002$ & $1.45294 \pm 0.00014$ \\
\hline PR2008 & $1540.297 \pm 0.020$ & $0.489 \pm 0.002$ & $1.45323 \pm 0.00014$ \\
\hline Special fibre & $1539.753 \pm 0.020$ & $0.092 \pm 0.002$ & $1.45262 \pm 0.00014$ \\
\hline
\end{tabular}

\section{Conclusions}

A method is presented for accurate determination of the effective refractive indices of optical fibres. The effective refractive indices of optical fibres at $\lambda \sim 1535 \mathrm{~nm}$ were measured for standard telecommunication fibres and for highly $\mathrm{GeO}_{2}$ doped photosensitive speciality fibres with an accuracy of $\sigma\left(n_{\text {eff }}\right) \sim 1.410^{-4}$. Differences in $\mathrm{GeO}_{2}$ concentrations were clearly resolved by the obtained values of the effective refractive indices. The effective refractive indices of different fibres with the same $\mathrm{GeO}_{2}$ concentrations were found to be very similar. A precise knowledge of $n_{\text {eff }}$ is important for the inscription of fibre Bragg gratings with precise wavelength specifications. This method can also have important applications in the process control for optical fibre manufacturing.

\section{Acknowledgements}

The authors would like to tank M. Rothhardt (IPHT, Jena, Germany) and C. Chojetzki (FBGS Technologies, Jena, Germany) for providing us with speciality photosensitive fibres. This work was supported by the German Minister of Research and Technology under AIF 1735X06.

\section{References}

[1] Hill, K. O., et al. "Photosensitivity in optical fiber waveguides: Application to reflection filter fabrication," Applied Physics Letters, Vol. 32, 1978, pp.647-649. 
[2] Othonos, A., Kalli, K., "Fiber Bragg Gratings: Fundamentals and Applications in Telecommunications and Sensing", Artech House Inc., Boston, London, 1999

[3] Hill, K. O. and Meltz, G., "Fiber bragg grating technology fundamentals and overview," Journal of Lightwave Technology, 15, 1263-1276 (1997).

[4] Kersey, A. D., Davis, M. A., Patrick, H. J., Koo, K. P., Askins C. G., Putnam, M. A. and Friebele, E. J., "Fiber grating sensors," Journal of Lightwave Technology, 15, 1442-1463 (1997).

[5] Hill, K. O., et al., "Fiber Bragg Gratings fabricated in monomode photosensitive optical fibers by UV exposure through a phase mask", Applied Physics Letters, 62, 1993, pp. 1035-1037

[6] Roths, J., Jülich, F., "Determination of strain sensitivity of free fibre Bragg gratings", Proc. SPIE 7003, 700308, (2008)

[7] Högenauer, D., "Herstellung von Faser-Bragg-Gittern," diploma thesis, Munich University of Applied Sciences (2006) (in German)

[8] ORMOCERE ${ }^{\circledR}$ (trademark of the Fraunhofer-Gesellschaft zur Förderung der angewandten Forschung e.V. München), Fraunhofer-Institut für Silicatforschung ISC, Würzburg, Germany

[9] Gilbert, S.L., Swann, W.C., Acetylene ${ }^{12} \mathrm{C}_{2} \mathrm{H}_{2}$ Absorption Reference for 1510 to 1540nm Wavelength Calibration - SRM 2517a, NIST Special Publication 260-133 (2001 Edition) 